\title{
Chronic Obstructive Pulmonary Disease: The Role of Myocardial Deformation Indices and Right Ventricle Three-Dimensional Echocardiography
}

\author{
Claudia Myriam Amaral Botelho, ${ }^{1}$ José Luiz Barros Pena, ${ }^{2,3}$ Bruno Rezende Passos, ${ }^{\circledR}$ Paulo Roberto Latorre \\ Fortes, $^{3 \odot}$ Maria da Consolação Vieira Moreira ${ }^{1}{ }^{\circledR}$ \\ Faculdade de Medicina da UFMG,' Belo Horizonte, MG - Brazil \\ Faculdade Ciências Médicas de Minas Gerais, ${ }^{2}$ Belo Horizonte, MG - Brazil \\ Hospital Felício Rocho, ${ }^{3}$ Belo Horizonte, MG - Brazil
}

\section{Abstract}

Background: Right ventricular dysfunction is a major complication in chronic obstructive pulmonary disease (COPD) and, despite its prognostic implications, is rarely considered in routine clinical analysis.

Objective: To compare RV function variables with standard and advanced echocardiographic techniques in patients with stable COPD and controls.

Methods: Twenty COPD patients classified as GOLD $\geq$ II (13 men aged $68.4 \pm 8.3$ years) and 20 matched controls were compared. Myocardial strain/strain rate indices were obtained by tissue Doppler and two-dimensional speckle tracking echocardiography. Right ventricular ejection fraction was obtained with three-dimensional software. Free wall myocardial thickness (FWMT) and tricuspid annular systolic excursion (TAPSE) were also measured. Numerical variables were compared between groups with Student's t-test or the Mann-Whitney test. Associations between categorical variables were determined with Fisher's exact test. P-values $<0.05$ were considered statistically significant.

Results: All myocardial deformation indices, particularly global longitudinal strain $(-17.2 \pm 4.4$ vs $-21.2: \pm 4.4=$ $0.001)$ and $3 \mathrm{D}$ right ventricular ejection fraction $(40.8 \pm 9.3 \%$ vs $51.1 \pm 6.4 \% \mathrm{p}<0.001)$ were reduced in COPD patients. These patients presented higher right ventricular FWMT and lower TAPSE values than controls.

Conclusion: Myocardial deformation indices, either tissue Doppler or speckle tracking echocardiography and 3D right ventricular ejection fraction, are robust markers of right ventricular dysfunction in patients with stable COPD. Assessing global longitudinal strain by speckle tracking echocardiography is a more practical and reproducible method.

Keywords: Pulmonary Disease Chronic Obstructive-COPD; Cardiomyopathies; Ventricular Dysfunction Right; Echocardiography, Three-Dimensional/ methods.

\section{Introduction}

Chronic obstructive pulmonary disease (COPD), considered a global epidemic, is currently the fourth leading cause of death worldwide. ${ }^{1,2}$ This public health problem is responsible for substantial morbidity and mortality, as well as high health care costs. Although defined as an inflammatory process that results in airway obstructions and parenchymal destruction, changes in pulmonary vessels are an important component of COPD and can cause right ventricular (RV) dysfunction, even in less severe patients. ${ }^{3}$ Despite their prognostic implications, ${ }^{4}$ changes in RV function are often overlooked in routine clinical assessments. In addition, $\mathrm{RV}$ analysis with conventional echocardiography is technically challenging in these cases, either due to complex geometry or the pulmonary hyperinflation that occurs in COPD patients. However, three-dimensional echocardiography and myocardial deformation indices 
seem to be effective, non-invasive, and reproducible tools for assessing function. ${ }^{5,6}$

This study's aim was to compare standard echocardiographic RV parameters with myocardial deformation indices and new three-dimensional (3D) echocardiography techniques in COPD patients and normal controls.

\section{Material and Methods}

This clinical, observational and case-control study was conducted at the COPD outpatient clinic of the Hospital das Clínicas, Federal University of Minas Gerais, Belo Horizonte, Brazil. It was reviewed and approved by the local ethics committee. All participants provided written informed consent.

\section{Population}

Twenty COPD patients and 20 controls matched for sex and age were included in the study, as illustrated in Figure 1. The inclusion criteria for all participants were: $\geq 40$ years of age, no history of asthma, and no known significant cardiovascular disease (especially coronary artery disease, heart failure, or complex arrhythmias). COPD patients had to be current or previous smokers with a history of at least 20 pack-years and airway obstruction classified as GOLD II-IV. The exclusion criteria were exacerbation in the previous 30 days and clinical signs of cor pulmonale.

\section{Methods}

This cross-sectional case-control study compared variables in two groups of individuals without known heart disease (with and without COPD), matched for sex and age ( \pm 5 years). COPD patients were stratified according to several isolated prognostic parameters and multidimensional indexes, observing the distribution of echocardiographic variables.

The sample size estimation was based on right ventricular function studies, including reference values from 2D and 3D echocardiographic parameters according to the most up-to-date international guidelines. ${ }^{7-9}$ After data collection, the sample power was calculated for the most important variables.

\section{Clinical and Laboratory Evaluation}

All participants underwent a complete clinical evaluation by a pulmonologist, including other reported health problems. Hemoglobin, fasting blood glucose,

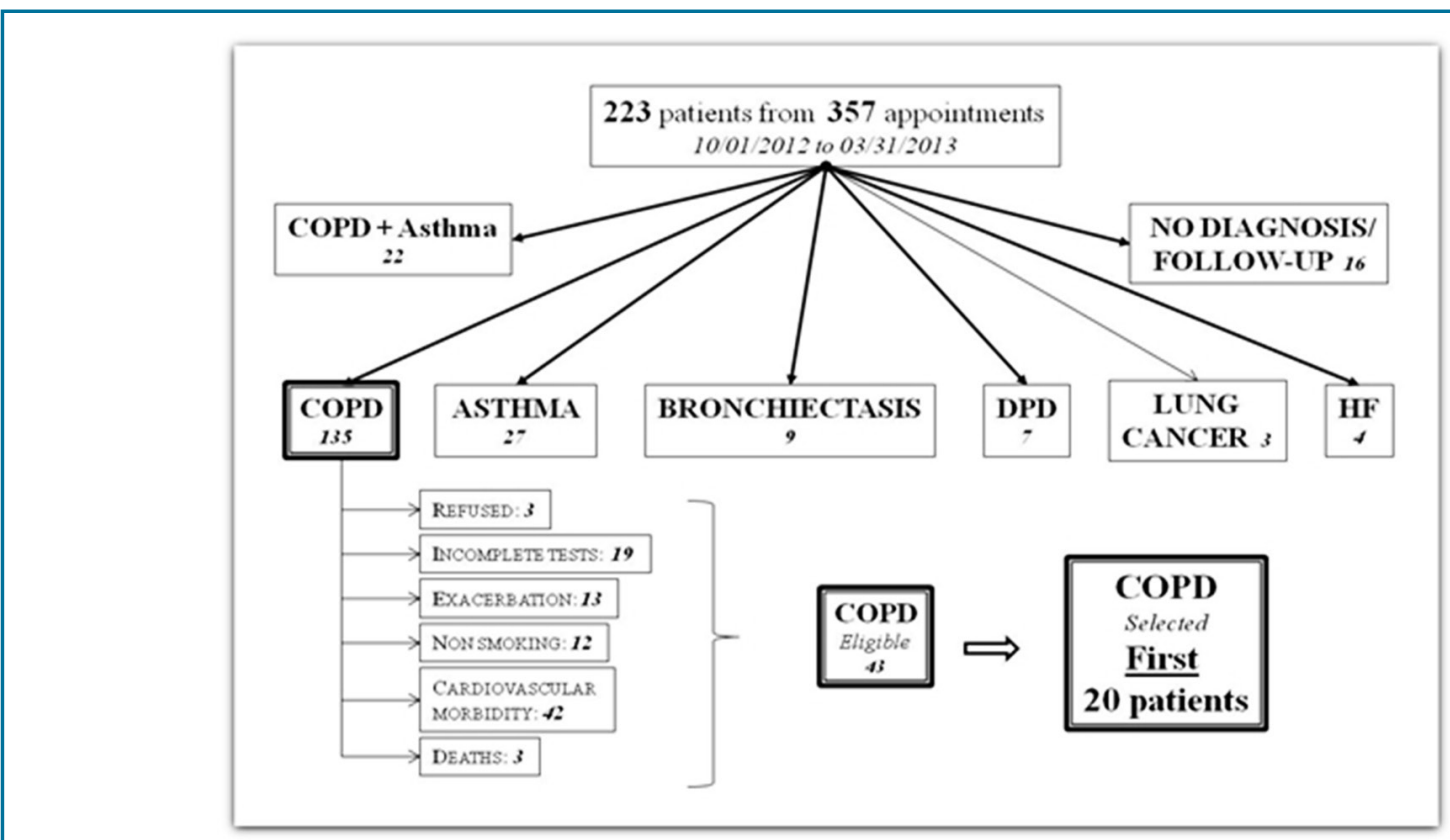

Figure 1 - Patient selection from the COPD Outpatient Clinic, Hospital das Clinicas, Federal University of Minas Gerais. COPD = chronic obstructive pulmonary disease; $\mathrm{DPD}=$ diffuse pulmonary disease; $\mathrm{HF}$ = heart failure. 
renal function tests, electrocardiography, and chest x-rays were obtained from all participants and were used to exclude other serious diseases.

\section{Pulmonary Function Tests}

For all participants, the same technician performed spirometry with a Koko spirometer (2002 Pulmonary Data Services Instrumentation, Louisville, CO, USA). The absolute values and percentages of post-bronchodilator forced expiratory volume in 1 second and vital capacity were obtained from all participants according to Brazilian guidelines and reference values. ${ }^{10}$ All participants performed the 6-minute walk test twice according to American Thoracic Society guidelines, ${ }^{11}$ and the greatest distance covered was used in the analysis.

\section{Echocardiography}

All participants underwent a comprehensive transthoracic echocardiographic examination according to American Society of Echocardiography recommendations ${ }^{9}$ using a Vivid 7 imaging system (GE Vingmed Ultrasound, Horten, Norway). For all parameters, the analysis considered the mean of three measurements taken by two experienced echocardiographers blinded to the patients' medical history.

The M-mode values provided 1D diameters (in millimeters) of the aorta, left atrium, right ventricle in diastole, left ventricle (LV) in diastole, and systole, and the inferior vena cava. The interventricular septum and LV posterior wall were also measured. RV free wall thickness was measured (in millimeters) at end diastole with 2D-oriented M-mode imaging in a subcostal view. Tricuspid annular plane systolic excursion (TAPSE) was obtained in M-mode, guided by a focused 2D apical 4-chamber examination. LV volumes at end diastole and systole and LV ejection fraction (EF) were measured using the Teichholz method (M-mode) to assess LV systolic function. LVEF was also obtained by a 2D examination using the Simpson method. RV linear measurements were taken using 2D imaging, including the RV basal dimension and RV mid-cavity from the apical 4-chamber view. RV dimension was also measured in the parasternal short and long axis views. Peak tricuspid regurgitant jet velocity and pulmonary artery systolic pressure were measured with color Doppler; continuous-wave Doppler was used in an attempt to obtain better alignment of the regurgitant jet.

Special adjustments were made to measure myocardial deformation values (strain/strain rate) in tissue Doppler.
To obtain the highest possible frame rate, $\leq 30^{\circ}$ reduction was used, as well as depth reduction. The tilt feature was used for better alignment to keep the RV wall at the center of the ultrasound sector.

Simultaneously, an appropriate adjustment of the speed range setting was used to prevent aliasing. Three complete consecutive cardiac cycles were observed in the apical 4-chamber view (emphasizing the RV) and were digitally recorded and stored. The frame rate for all participants was $\geq 150 /$ s. The digitized images were transferred to a computer and analyzed using Echo PAC GE software (PCTM by SW, version 110.1.0; Vingmed Ultrasound Horten, Norway).

Global longitudinal strain (GLS) was measured with speckle tracking using 2D image motion in the apical fourchamber view according to current recommendations. ${ }^{7,8}$ Three cardiac cycles were obtained in the RV-focused apical four-chamber window. We attempted to obtain the best image without interference, using a frame rate of 60-80/s. GLS was measured using a 6-segment RV model (including interventricular septum and RV free wall). The data were sent to a separate workstation (EchoPAC version 112.1.3., GE Healthcare) for further offline analysis.

For data acquisition, real-time 3D echocardiograms were used with the full-volume pattern, which was obtained with a matrix-array transducer. Post-processing analyses (4D RV-FUNCTION; TomTec Imaging Systems $\mathrm{GmbH}$, Unterschleissheim, Germany) were performed with semi-automatic software, which traced the predefined RV borders to detect the endocardial contour. This allowed measurement of the RV end-diastole, RV end-systole, and RV stroke volume (RVSV).

\section{Statistical Analysis}

Statistical analyses were performed using IBM SPSS Statistics version 18 (IBM, Armonk, NY, USA). Categorical variables were expressed as frequencies. Continuous and normally-distributed variables were expressed as means and standard deviations (SD) and compared using Student's t-test for independent samples. Non-normally distributed variables were compared using the Mann-Whitney test and were expressed as median ( $1^{\text {st }} ; 3^{\text {rd }}$ quartiles). The normality of the variables was assessed with the Shapiro-Wilk test. Associations between categorical variables were determined with Fisher's exact test. $\mathrm{P}<0.05$ was considered statistically significant for all measurements. 
The echocardiographic values for 10 variables from 5 randomly chosen COPD patients and 5 controls were reanalyzed by both observers $\geq 20$ days after the first evaluation. These patients were selected by randomly drawing numbered slips of paper representing the two exam groups, without comparing them to previous results. Means and SDs were obtained for every patient based on two measurements of each variable. Intra- and interobserver variability were then determined as a percentage of the mean $\pm \mathrm{SD}$ of all results for each variable. Bland-Altman plots were also used for GLS and RVEF.

\section{Results}

\section{Demographic Characteristics}

The general characteristics of the patients with stable COPD and respective controls are shown in Table 1.
The sample consisted primarily of older men. No participants presented comorbidities. The weight difference was smaller in the COPD group than the control group $(63.0 \pm 12.3 \mathrm{vs} 70.6 \pm 10.8 \mathrm{~kg}, p=0.046)$. Total pack-years and several comorbidities were predictably higher in the COPD group. There were no significant differences between the groups for the other general characteristics.

\section{M-Mode, 2D, and Doppler Echocardiography}

Table 2 compares the M-mode, 2D, and Doppler echocardiography results for the COPD and control groups.

The only M-mode parameters that differed significantly between the groups were TAPSE and RV free wall thickness. Only $30 \%$ of the COPD group had a normal

Table 1 - Demographic characteristics of 20 COPD patients and 20 controls

\begin{tabular}{|c|c|c|c|}
\hline & Patients & Controls & $P$-value \\
\hline \multicolumn{4}{|l|}{ Demographic variables } \\
\hline Age $^{2}$ (years) & $68.4 \pm 8.3$ & $67.6 \pm 9.3$ & 0.760 \\
\hline $\operatorname{Sex}^{1} \mathrm{M} / \mathrm{F}$ & $13 / 7$ & $13 / 7$ & 1.000 \\
\hline $\mathrm{BMI}^{2}\left(\mathrm{~kg} / \mathrm{m}^{2}\right)$ & $24.4 \pm 4.2$ & $26.7 \pm 3.2$ & 0.050 \\
\hline Packs/year ${ }^{3}$ & $51.5(35.8 ; 60.0)$ & $2.5(0.0 ; 19.5)$ & 0.000 \\
\hline Current smoker ${ }^{1}$ & $4(20)$ & $3(15)$ & 0.681 \\
\hline $\mathrm{SBP}^{2}(\mathrm{mmHg})$ & $128 \pm 11$ & $133 \pm 11$ & 0.182 \\
\hline $\mathrm{DBP}^{2}(\mathrm{mmHg})$ & $77 \pm 9$ & $81 \pm 6$ & 0.135 \\
\hline Comorbidities/person & $3.5 \pm 1.4$ & $2.5 \pm 1.5$ & 0.025 \\
\hline Cardiovascular $^{1}(\mathrm{n})$ & $17(85)$ & $10(50)$ & 0.043 \\
\hline Respiratory $^{1}(\mathrm{n})$ & $13(65)$ & $12(60)$ & 1.000 \\
\hline Gastrointestinal $^{1}(\mathrm{n})$ & $10(50)$ & $9(45)$ & 1.000 \\
\hline Metabolic $^{1}(\mathrm{n})$ & $22(110)$ & $20(100)$ & 1.000 \\
\hline Genitourinary $^{1}(\mathrm{n})$ & $1(5)$ & $5(25)$ & 0.184 \\
\hline Neuropsychiatry $^{1}(\mathrm{n})$ & $6(30)$ & $3(15)$ & 0.449 \\
\hline \multicolumn{4}{|l|}{ Laboratory tests } \\
\hline Hemoglobin $^{2}(\mathrm{~g} / \mathrm{dl})$ & $14.5 \pm 1.7$ & $14.7 \pm 1.2$ & 0.701 \\
\hline Glucose level ${ }^{2}$ (mg/dl) & $103.0 \pm 29.3$ & $98.5 \pm 20.0$ & 0.718 \\
\hline Creatinine level$^{2}(\mathrm{mg} / \mathrm{dl})$ & $0.92 \pm 0.22$ & $0.93 \pm 0.22$ & 0.960 \\
\hline
\end{tabular}

Data are presented as: (1) absolute number ( $n)$ - Fisher's test; (2) mean $\pm S D$-Student's $t$-test; (3) median (1 ${ }^{\text {st; }} 3^{\text {rd }}$ quartile) - Mann-Whitney test. BMI: body mass index; M: male; F: female; SBP: systolic blood pressure; DBP: diastolic blood pressure. 
Table 2 - Conventional echocardiography variables in COPD patients and controls

\begin{tabular}{|c|c|c|c|}
\hline Variables & Patients & Controls & $P$-value \\
\hline \multicolumn{4}{|l|}{ M mode } \\
\hline $\mathrm{Ao}^{1}(\mathrm{~mm})$ & $34.9 \pm 4.2$ & $33.9 \pm 3.2$ & 0.429 \\
\hline $\mathrm{LA}^{1}(\mathrm{~mm})$ & $34.1 \pm 4.2$ & $36.6 \pm 3.8$ & 0.050 \\
\hline $\operatorname{RVDd}^{1}(\mathrm{~mm})$ & $18.7 \pm 5.8$ & $19.3 \pm 3.4$ & 0.718 \\
\hline $\mathrm{RVWt}^{1}(\mathrm{~mm})$ & $6.50 \pm 1.7$ & $4.40 \pm 0.53$ & $<0.001$ \\
\hline $\mathrm{LVs}^{1}(\mathrm{~mm})$ & $28.1 \pm 4.1$ & $29.3 \pm 2.2$ & 0.259 \\
\hline $\mathrm{LVd}^{1}(\mathrm{~mm})$ & $45.5 \pm 5.2$ & $46.2 \pm 4.0$ & 0.610 \\
\hline $\operatorname{IVS}^{2}(\mathrm{~mm})$ & $8.6(7.6 ; 9.4)$ & $8.2(8.0 ; 8.7)$ & 0.363 \\
\hline $\mathrm{PW}^{2}(\mathrm{~mm})$ & $8.2(7.4 ; 9.1)$ & $8.2(8.0 ; 8.8)$ & 0.924 \\
\hline $\mathrm{LVD}^{2}(\mathrm{~mL})$ & $94.5(82.0 ; 113.3)$ & $88.0(82.8 ; 110.8)$ & 0.850 \\
\hline $\mathrm{LVS}^{1}(\mathrm{~mL})$ & $30.8 \pm 10.1$ & $32.2 \pm 7.7$ & 0.639 \\
\hline $\mathrm{LVSv}^{2}(\mathrm{~mL})$ & $66.0(57.5 ; 79.5)$ & $59.5(54.8 ; 78.5)$ & 0.457 \\
\hline $\operatorname{TAPSE}^{1}(\mathrm{~mm})$ & $18.0 \pm 3.7$ & $21.5 \pm 3.1$ & 0.002 \\
\hline $\operatorname{LVEFT}^{1}(\%)$ & $68.9 \pm 6.4$ & $66.3 \pm 4.4$ & 0.142 \\
\hline \multicolumn{4}{|c|}{ Two-dimensional echocardiography } \\
\hline LVEFS $^{1}(\%)$ & $58.5 \pm 4.5$ & $56.6 \pm 4.4$ & 0.190 \\
\hline $\mathrm{RVDb}^{1} \mathrm{~A} 4 \mathrm{C}(\mathrm{mm})$ & $37.3 \pm 6.8$ & $34.3 \pm 4.1$ & 0.100 \\
\hline $\mathrm{RVDm}^{1} \mathrm{~A} 4 \mathrm{C}(\mathrm{mm})$ & $28.1 \pm 5.9^{\S}$ & $27.1 \pm 4.0$ & 0.510 \\
\hline $\operatorname{RVDPSAX}^{2}(\mathrm{~mm})$ & $26.8(23.0 ; 30.0)^{\S}$ & $24.7(23.0 ; 26.0)$ & 0.140 \\
\hline $\operatorname{RVDPLAX}^{2}(\mathrm{~mm})$ & $26.5(25.0 ; 30.7)$ & $26.5(24.8 ; 29.0)$ & 0.470 \\
\hline \multicolumn{4}{|c|}{ Doppler Echocardiography ${ }^{\S}$} \\
\hline $\operatorname{TRPV}^{1}(\mathrm{~m} / \mathrm{s})$ & $2.90 \pm 0.38$ & $2.25 \pm 0.16$ & $<0.001$ \\
\hline $\operatorname{PASP}^{1}(\mathrm{~mm} \mathrm{Hg})$ & $43.2 \pm 11.02$ & $25.4 \pm 2.87$ & $<0.001$ \\
\hline \multicolumn{4}{|c|}{ 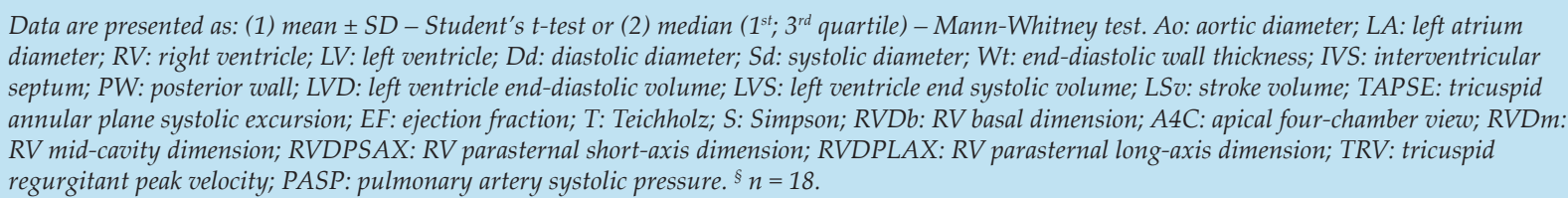 } \\
\hline
\end{tabular}

RV free wall thickness (considering $5 \mathrm{~mm}$ as the cut-off point). All LV anatomical and functional parameters in M-mode were within normal limits and were similar between groups. In 2D echocardiography, the linear dimensions of the RV did not differ significantly between the COPD and control groups. However, patients with lung disease had significantly higher peak regurgitant jet velocity and pulmonary artery systolic pressure values than controls.

\section{Myocardial Deformation and Three-Dimensional Echocardiography}

RVEF and RVSV were significantly lower in the COPD group. All deformation indices (strain/strain rate) were significantly lower in all segments in the COPD group, which indicates reduced regional deformation that affects the RV free wall. GLS also differed significantly between the groups, indicating RV dysfunction in the COPD group (Figure 2 and Table 3). 


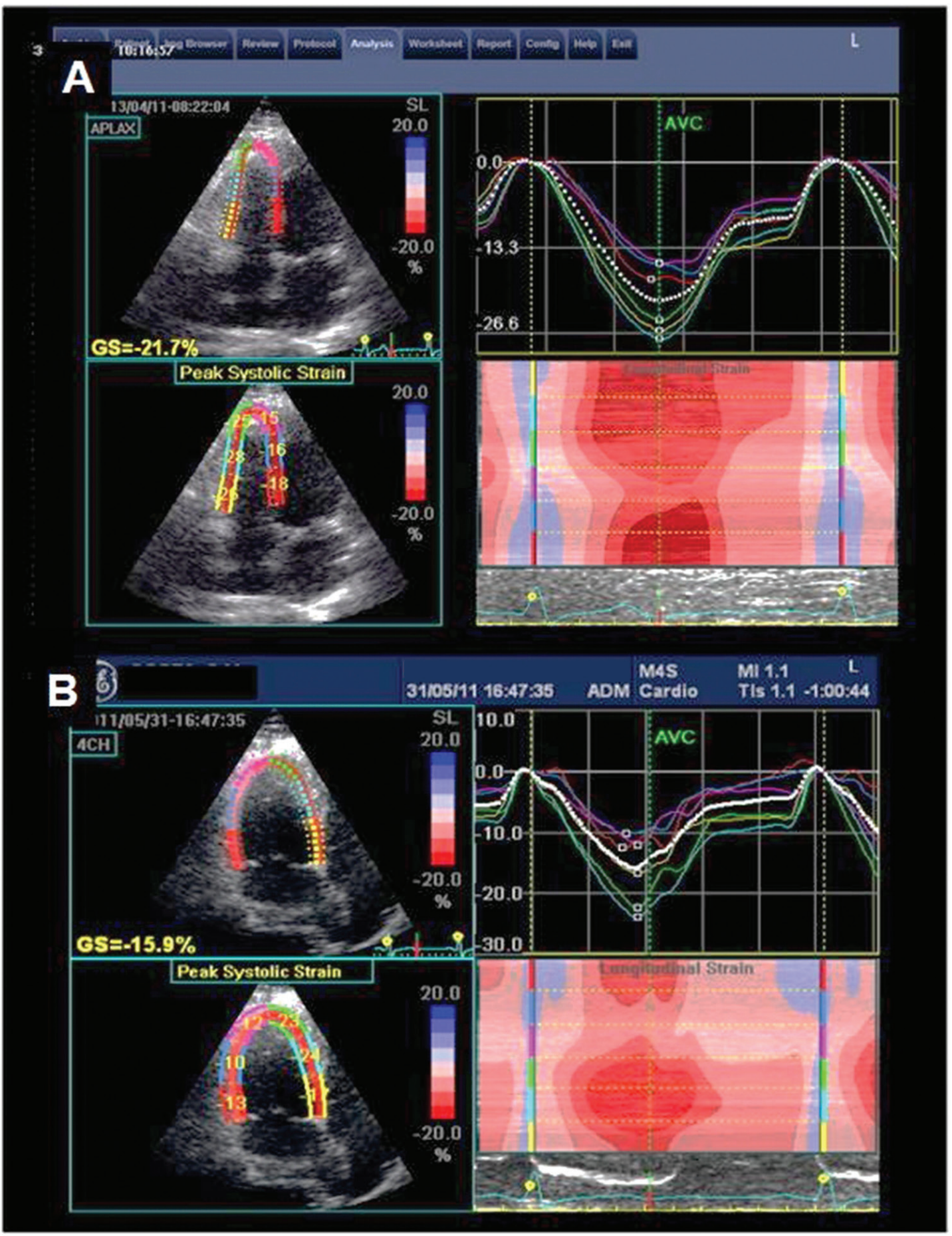

Figure 2 - 2D speckle tracking analysis from a right ventricle-focused apical 4-chamber view. The mean global systolic strain values and time curves were obtained by tracking a 6-segment ROI. In A, a patient from the control group, the GLS was -21.7\%, while in B, a patient from the COPD group, it was $-\mathbf{1 5 . 9 \%}$.

ROI: region of interest; GLS: global longitudinal strain 
Table 3 - Myocardial deformation indices and three-dimensional echocardiography in COPD patients and controls

\begin{tabular}{|c|c|c|c|}
\hline Variables & Patients & Controls & $P$-value \\
\hline \multicolumn{4}{|l|}{ Strain by TDI (\%) } \\
\hline $\mathrm{Sb}^{2}$ & $-19.0(-26.6 ;-14.2)$ & $-23.9(-31.1 ;-19.4)$ & 0.015 \\
\hline $\mathrm{S} \mathrm{m}^{2}$ & $-19.6(-23.5 ;-16.0)$ & $-28.2(-30.3 ;-18.2)$ & 0.033 \\
\hline $\mathrm{Sa}^{1}$ & $-17.3 \pm 6.3$ & $-24.3 \pm 6.3^{\S}$ & 0.002 \\
\hline \multicolumn{4}{|c|}{ Strain rate by TDI $\left(\mathrm{s}^{-1}\right)$} \\
\hline $\mathrm{SR} \mathrm{b}^{1}$ & $-1.35 \pm 0.44$ & $-1.75 \pm 0.45$ & 0.005 \\
\hline $\mathrm{SR} \mathrm{m}^{1}$ & $-1.44 \pm 0.49$ & $-1.78 \pm 0.43$ & 0.025 \\
\hline $\mathrm{SR} \mathrm{a}^{1}$ & $-1.27 \pm 0.42$ & $-1.66 \pm 0.52^{\S}$ & 0.016 \\
\hline \multicolumn{4}{|c|}{ 2D strain by speckle tracking (\%) } \\
\hline GLS $^{1}$ & $-17.2 \pm 4.4$ & $-21.2 \pm 2.0 \$ \$$ & 0.001 \\
\hline \multicolumn{4}{|c|}{ 3D echocardiography ${ }^{\oplus}$} \\
\hline $\operatorname{RVDv}(\mathrm{mL})^{1}$ & $59.9 \pm 19.7$ & $62.3 \pm 13.6$ & 0.658 \\
\hline RVeSv $(m L)^{1}$ & $35.0 \pm 13.0$ & $30.9 \pm 7.4$ & 0.240 \\
\hline $\operatorname{RVEF}(\%)^{1}$ & $40.8 \pm 9.3$ & $51.1 \pm 6.4$ & $<0.001$ \\
\hline $\operatorname{RVSv}(m L)^{1}$ & $24.8 \pm 10.1$ & $32.3 \pm 8.9$ & 0.022 \\
\hline \multicolumn{4}{|c|}{$\begin{array}{l}\text { Data are presented as: (1) mean } \pm S D-\text { Student's } t \text {-test; }(2) \text { median }\left(1^{\text {st }} ; 3^{\text {rd }} \text { quartile }\right) \text { - Mann-Whitney test. }{ }^{\S} \text { Data from } 18 \text { controls; } \$ \$ \text { data from } 19 \\
\text { controls; } \$ \text { data from } 17 \text { COPD patients and } 20 \text { controls. S: strain; SR: strain rate; TDI: tissue Doppler imaging; GLS: global longitudinal strain; b: basal, } \\
\text { m: medium; a: apical. } 2 D \text { : two-dimensional; } 3 D \text { : three-dimensional; RV: right ventricular; Dv: end-diastolic volume; eSv: end-systolic volume; } \\
\text { EF: ejection fraction; Sv: stroke volume. }\end{array}$} \\
\hline
\end{tabular}

The RVEF difference between groups was highly significant. RVSV also differed significantly between the groups, showing that 3D echocardiogram can identify RV dysfunction in stable COPD patients. (Figure 3 and Table 3 ).

Only one COPD patient (5\%) showed no changes related to the significantly different echocardiographic variables (RV end-diastole, TAPSE, pulmonary artery systolic pressure, GLS, and RVEF). Two patients (10\%) differed in only one parameter (one with reduced GLS, the other with reduced RVEF). Nine other patients (45\%) showed changes in either four or five of these variables.

\section{Variability}

Table 4 shows the intra- and interobserver variability for the main variables. Figure 4 shows the Bland-Altman plots for RV functional variables, GLS, and RVEF.

\section{Discussion}

According to our results, echocardiographic parameters of RV anatomy and function, mainly 2D
GLS and 3D RVEF, can identify RV systolic dysfunction in patients with stable, moderate-to-severe COPD. ${ }^{12}$

$\mathrm{RV}$ function assessment continues to be a challenge due to its complex geometry and location, narrow acoustic window, and a lack of quantitative, easy, and reproducible functional methods. ${ }^{13}$ Moreover, RV shape and performance depend on extrinsic factors such as preload, afterload, and LV function. ${ }^{14-16}$ Thus, the search continues for new modalities that can produce more reliable RV data than current conventional echocardiographic techniques. ${ }^{17-20}$

RV failure has prognostic implications in COPD patients, ${ }^{14,21}$ and its prevalence is estimated at around $10-30 \%$ for this group. In COPD pathogenesis, RV dysfunction occurs prior to signs and symptoms of systemic venous congestion. ${ }^{14}$

RV strain has emerged as the most accurate and sensitive echocardiographic tool for assessing RV function, with excellent subclinical detection of RV dysfunction. ${ }^{7,22,23}$ Longitudinal strain, calculated by both tissue Doppler and speckle-tracking techniques (both of 


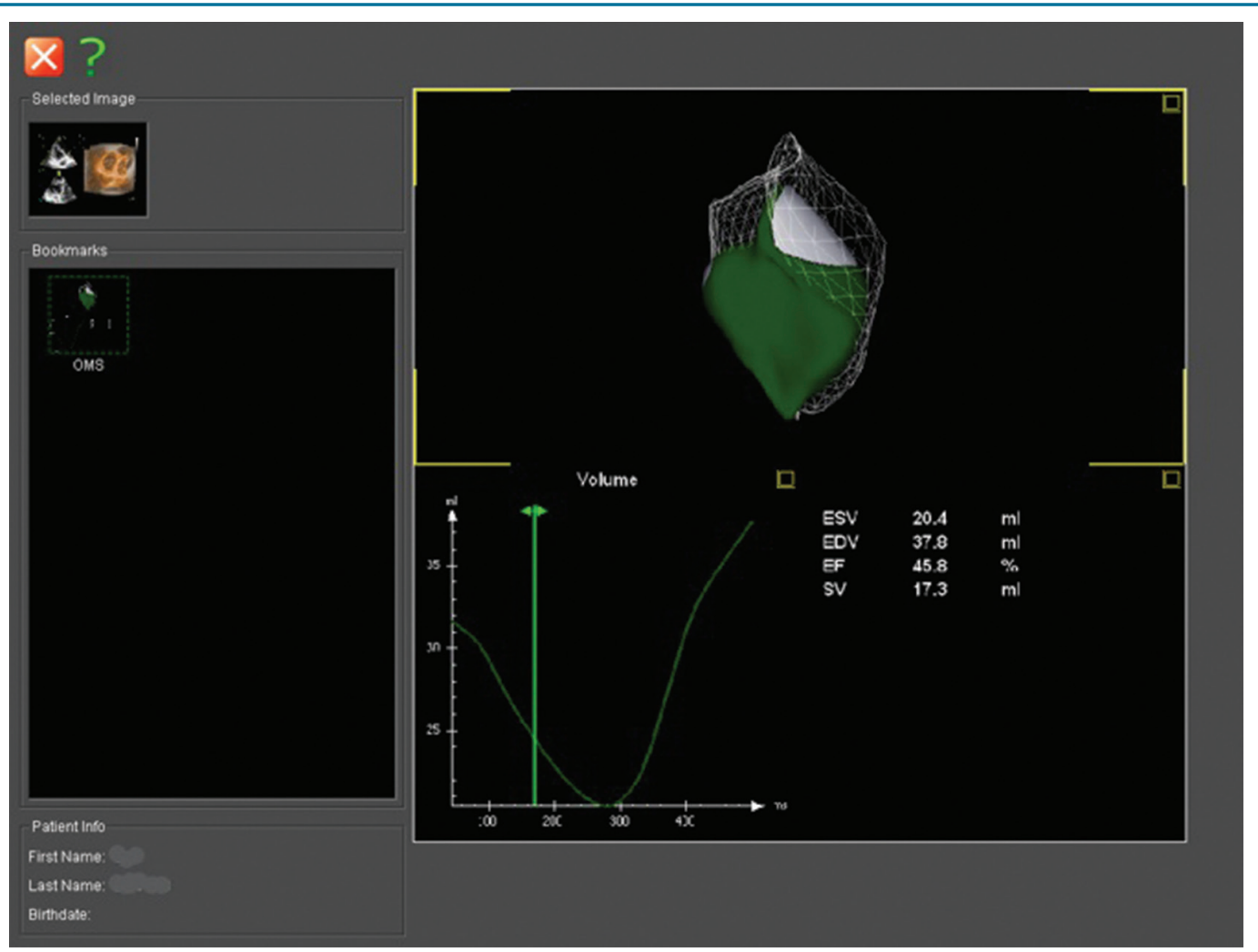

Figure 3 - The 3D dataset was acquired from an RV-focused, apical 4-chamber view. The image shows the 3D RV model with the volume curve.

EDV: end diastolic volume; EF: ejection fraction; ESV: end systolic volume; RV: right ventricle; SV: stroke volume.

which have been validated by magnetic resonance $)^{5,24}$ has also proven to be a reliable and reproducible instrument for analyzing RV function, with good correlations between clinical and invasive parameters and low intraand interobserver variability. ${ }^{7}$ Currently, the speckletracking technique is considered the method of choice for calculating GLS, since it is angle independent, more reproducible, and has lower inter- and intraobserver variability.

Our results showed that regional and global RV contractility, quantified as strain/strain rate values and 2D strain, were significantly lower in COPD patients than controls, in agreement with the literature..$^{24,25}$ We found GLS to be the strongest predictor of RV dysfunction in COPD patients. ${ }^{26}$

It should be pointed out that reduced myocardial deformation occurred in all RV segments, since Dopplerbased regional systolic strain was demonstrated. GLS measurement is a faster, more reliable, and reproducible technique. The reference values have recently been published. ${ }^{27}$
In the present study, 3D RVEF and 3D RVSV were robust markers of RV systolic dysfunction between COPD patients and controls. Real-time 3D echocardiography imaging overcomes the potential limitations of 2D techniques. ${ }^{28}$ It provides quantification of RV volumes and functional assessments without geometric hypotheses and uses commercially available software. Following current guidelines, a reference limit of $\leq 44 \%$ for RVEF was used to indicate dysfunction. ${ }^{9}$ The main limitation of this method is acquiring highquality 3D images in "full volume". We were unable to measure RV volumes in $3(15 \%)$ of 20 COPD patients due to technical difficulties. We also found that RVDV and RVSV had greater interobserver variability, which was previously mentioned by Aunee et al., ${ }^{29}$ when attempting to establish reference intervals for RV volumes.

\section{Limitations}

This study has some limitations. When selecting COPD patients, no specific exams were performed to exclude 
Table 4 - Intra- and interobserver variability (\%)

\begin{tabular}{|c|c|c|c|c|}
\hline \multirow{2}{*}{ Variable } & \multicolumn{2}{|c|}{ Intraobserver } & \multicolumn{2}{|c|}{ Interobserver } \\
\hline & Mean & $S D$ & Mean & $S D$ \\
\hline TAPSE & 5.4 & 2.4 & 5.5 & 4.2 \\
\hline $\mathrm{RVDb}$ & 3.4 & 2.7 & 5.0 & 3.0 \\
\hline RVDm & 3.9 & 3.1 & 5.6 & 7.5 \\
\hline RVesv & 3.7 & 5.5 & 10.7 & 9.8 \\
\hline $\mathrm{RVdv}$ & 4.2 & 2.5 & 8.7 & 8.2 \\
\hline RVsv & 6.7 & 5.0 & 12.2 & 9.2 \\
\hline RVEF & 3.6 & 3.2 & 5.5 & 3.7 \\
\hline $\mathrm{Sb}$ & 6.6 & 4.3 & 7.6 & 6.1 \\
\hline $\mathrm{Sm}$ & 6.4 & 4.3 & 8.9 & 5.6 \\
\hline GLS & 4.4 & 2.4 & 3.5 & 4.9 \\
\hline
\end{tabular}

other causes of RV dysfunction (such as pulmonary thromboembolism or subclinical coronary artery disease), although no clinical, electro-, or echocardiographic evidence of these diseases was found.

Two-dimensional strain involves some limitations that should be considered ${ }^{7}$. The analysis software was designed to evaluate LV systolic function and was adapted for the RV. The RV chamber has a thin wall and a more complex shape than the LV, with different inlet and outlet different planes. These anatomical features complicate selecting the width of the region of interest and tracking speckles frame by frame.

Although our sample of COPD patients was too small to draw generalized conclusions, the study could generate new research questions and propaedeutic investigations. The current literature on determining RV function through deformation indices in COPD patients is scarce.

\section{Conclusion}

Myocardial deformation indices and 3D echocardiographic parameters related to RV function analysis are reduced in stable COPD patients. The measurements provide a safe, reliable, reproducible, and non-invasive alternative for analyzing their subclinical RV changes. Routine inclusion of echocardiographic parameters when assessing these patients could provide a better understanding the natural history of cardiovascular complications in this disease. Incorporating these measurements into multidimensional COPD prognostic indices might lead to better therapeutic strategies and improve patient life expectancy.

\section{Potential Conflict of Interest}

No potential conflict of interest relevant to this article was reported.

\section{Sources of Funding}

There were no external funding sources for this study.

\section{Study Association}

This article is part of the thesis of Doctoral submitted by Claudia Myriam Amaral Botelho, from Universidade Federal de Minas Gerais.

\section{Ethics approval and consent to participate}

This study was approved by the Ethics Committee of the UFMG/COEP under the protocol number 0051.0.2023.000-11. All the procedures in this study were in accordance with the 1975 Helsinki Declaration, updated in 2013. Informed consent was obtained from all participants included in the study. 

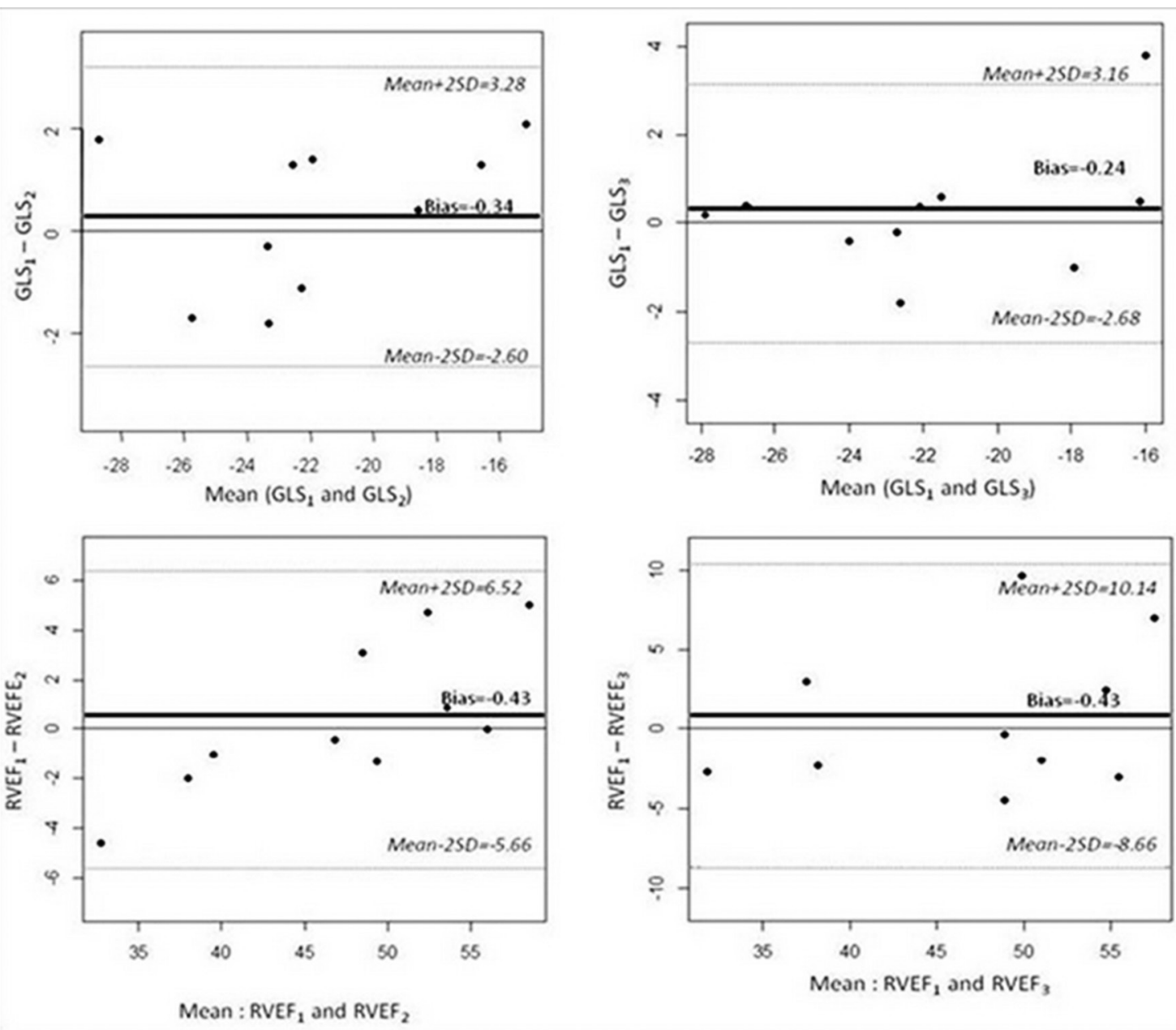

Figure 4 - Intraobserver (measurements 1 and 2) and interobserver (measurements 1 and 3) agreement for the 2D GLS and RVEF variables.

GLS: global longitudinal strain; RVEF: right ventricular ejection fraction.

\section{Author contributions}

Conception and design of the research: Botelho CMA, Pena JLB, Moreira MCV. Analysis and interpretation of the data: Botelho CMA, Pena JLB, Moreira MCV, Passos

\section{References}

1. Global Initiative for Chronic Obstructive Lung Disease (GOLD). Global Strategy for the Diagnosis, Management and Prevention of COPD . New Jersey;2017. [ Internet ] [Cited in 2021 May 12] Available from: http:// goldcopd.org.

2. Stirbulov R, Jardim JR. Diretrizes brasileiras para o manejo da DPOC. 2016. [Citado em 2020 Nov 23] Disponível em: www.sbpt.org.br

3. Tannus-Silva DG, Rabahi MF. State of the art Review of the Right Ventricle in COPD Patients: It is Time to Look Closer. Lung.2017 Feb;195(1):9-17.

4. Gökdeniz T; Kalaycıoğlu E, Boyacı, F Aykan, AÇ Gürsoy, MO, Hatem, et al. The BODE Index, a Multidimensional Grading System,
BR, Fortes PRL. Statistical analysis: Botelho CMA, Pena JLB, Passos BR. Writing of the manuscript: Botelho CMA, Pena JLB, Passos BR, Moreira MCV. Critical revision of the manuscript for intellectual content: Pena, JLB, Moreira MCV, Fortes PRL, Botelho CMA.

Reflects Impairment of Right Ventricle Functions in Patients with Chronic Obstructive Pulmonary Disease: A Speckle-Tracking Study. Respiration.2014;88(3):223-33.

5. Focardi M, Cameli M, Carbone SF, Massoni A, De Vito R, Lisi M, Mondillo, S. Traditional and innovative echocardiographic parameters for the analysis of right ventricular performance in comparison with cardiac magnetic resonance. Eur Heart J Cardiovasc Imaging.2015;16(1):47-52.

6. Atsumi A, Seo Y, Ishizu T, Nakamura A, Enomoto Y, Harimura $Y$ et al. Right Ventricular Deformation Analyses Using a Three-Dimensional 
Speckle-Tracking Echocardiographic System Specialized for the Right Ventricle. J Am Soc Echocardiogr.2016;29(5):402-11.

7. Longobardo L, Suma V, Jain R, Carerj S, Zito C, Zwicke DL et al. Role of Two-Dimensional Speckle-Tracking Echocardiography Strain in the Assessment of Right Ventricular Systolic Function and Comparison with Conventional Parameters. J Am Soc Echocardiogr. 2017;30(10):937-46.

8. Collier P, Phelan D, Klein A. A Test in Context, Myocardial Strain Measured by Speckle-Tracking Echocardiography. J Am Coll Cardiol. 2017;69(8):1043-56.

9. Lang RM, Badano LP, Mor-Avi V, Afilalo J, Armstrong A, Ernande L et al. Recommendations for cardiac chamber quantification by echocardiography in adults: an update from the American Society of Echocardiography and the European Association of Cardiovascular Imaging. J Am Soc Echocardiogr.2015;28(1):1-39.

10. Pereira CAC, Jansen JM, Barreto SSM, Marinho J, Sulmonett N, Dias RM. Espirometria. In: Diretrizes para testes de função pulmonar. J Pneumol 2002;28:S1-S82.

11. ATS Committee on Proficiency Standards for Clinical Pulmonary Function Laboratories. ATS Statement: Guidelines for Six-Minute Walk Test. Am J Respir Crit Care Med. 2002 Jul 1;166(1):111-7.

12. Hilde JM, Skjørten I, Grøtta OJ, Hansteen V, Melsom MN, Hisdal J et al. Right ventricular dysfunction and remodeling in COPD without pulmonar hypertension. J Am Coll Cardiol. 2013 Sep 17;62(12):1103-11.

13. Di Lorenzo MP, Bhatt SM, Mercer-Rosa L. How best to assess right ventricular function by echocardiography. Cardiol Young. 2015 Dec;25(8):1473-81.

14. Tayyareci Y, Tayyareci G, Tastan CP, Bayazit P, Nisanci Y. Early Diagnosis of Right Ventricular Systolic Dysfunction by Tissue Doppler-derived Isovolumic Myocardial Acceleration in Patients with Chronic Obstructive Pulmonary Disease. Echocardiography. 2009 Oct;26(9):1026-35.

15. Vandenheuvel MA, Bouchez S, Wouters PF, De Hert SG. A pathophysiological approach towards right ventricular function and failure. Eur J Anaesthesiol. 2013 Jul;30(7):386-94.

16. Vonk-Noordegraaf A, Westerhof N. Describing right ventricular function. Eur Respir J. 2013, 41:1419-23.

17. Kossaify A. Echocardiographic Assessment of the Right Ventricle, from the Conventional Approach to Speckle Tracking and Three-Dimensional Imaging, and Insights into the "Right Way" to Explore the Forgotten Chamber. Clin Med Insights Cardiol.2015 Jul 5;9:65-75.

18. Meris A, Faletra F, Conca C, Klersy C, Regoli F, Klimusina J et al. Timing and magnitude of regional right ventricular function: a speckle tracking-derived strain study of normal subjects and patients with right ventricular dysfunction. J Am Soc Echocardiogr.2010;23(8):823-31.

19. Cameli M, Righini FM, Lisi M, Mondillo S. Right ventricular strain as a novel approach to analyze right ventricular performance in patients with heart failure. Heart Fail Rev. 2014 Sep;19(5):603-10.

20. Chang WT, Liu YW, Liu PY, Hsu CH, Tsai WC. The association between right ventricular free wall strain and exercise capacity for health check-up subjects. MacGowan GA, ed. PLoS ONE. 2017;12(3):e0173307.

21. Tanaka Y, Hino M, Mizuno K, Gemma A. Evaluation of Right Ventricular Function in Patients With COPD. Respir Care. 2013 May;58(5):816-23.

22. Rice JL, Stream AR, Fox DL, Geraci MW, Vandivier RW, Dorosz JL et al. Speckle Tracking Echocardiography to evaluate for pulmonary hypertension in Chronic Obstructive Pulmonary Disease. COPD. 2016 Oct;13(5):595-600.

23. Sabit R, Bolton C, Fraser AG, Edwards J, Edwards PH, Ionescu AA, Cockcroft J, Shale DJ. 2010. Sub-clinical left and right ventricular dysfunction in patients with COPD. Respir Med.2010;104(8):1171-8.

24. Lu KJ, Chen JX, Profitis K, Kearney LG, De Silva D, Smith G, et al. Right Ventricular Global Longitudinal Strain Is an Independent Predictor of Right Ventricular Function: A Multimodality Study of Cardiac Magnetic Resonance Imaging, Real-Time Three-Dimensional Echocardiography and Speckle Tracking Echocardiography. Echocardiography. 2015 Jun;32(6):966-74

25. Liu Y, Wang D, Du Q, Che G, Tian J, Su Y. Evaluation of Right Ventricular Systolic Function in Patients with Chronic Pulmonary Heart Disease by 2-Dimensional Speckle-Tracking Echocardiography. J Ultrasound Med.2016 Nov;35(11):2333-42.

26. Schoos, MM. Echocardiographic predictors of exercise capacity and mortality in chronic obstructive pulmonary disease. BMC Cardiovasc Disord. 2013;13:84.

27. Muraru D, Onciul S, Peluso D, Soriani N, Cucchini U, Aruta P, et al Sex- and method-specific reference values for right ventricular strain by 2-dimensional speckle-tracking echocardiography. Circ Cardiovasc Imaging 2016;9:e003866.

28. Vitarelli A, Mangieri E, Terzano C, Gaudio C, Salsano F, Rosato E, Capotosto et al. Three-Dimensional Echocardiography and 2D-3D Speckle-Tracking Imaging in Chronic Pulmonary Hypertension: Diagnostic Accuracy in Detecting Hemodynamic Signs of Right Ventricular (RV) Failure. J Am Heart Assoc.2015 Mar 19;4(3):e001584.

29. Aune E, Baekkevar M, Rodevand O, Otterstad JE. Reference values for left ventricular volumes with real-time 3-dimensional echocardiography. Scand Cardiovasc J. 2010;44(1):24-30. 